\title{
First Isolation and Phylogenetic Analyses of Tick-Borne Encephalitis Virus in Lower Saxony, Germany
}

\author{
Mathias Boelke ${ }^{1,2, \dagger}$, Malena Bestehorn ${ }^{3,4,+}$, Birgit Marchwald ${ }^{5}$, Mareike Kubinski 1,2, \\ Katrin Liebig $^{1,2}$, Julien Glanz ${ }^{1,2}$, Claudia Schulz ${ }^{1,2}$, Gerhard Dobler ${ }^{3,4}$, Masyar Monazahian 5 \\ and Stefanie C. Becker 1,2,* \\ 1 Institute for Parasitology, University of Veterinary Medicine Hannover, Bünteweg 17, \\ 30559 Hanover, Germany; mathias.boelke@tiho-hannover.de (M.B.); mareikekub@gmail.com (M.K.); \\ Katrin.Liebig@tiho-hannover.de (K.L.); Julien.Glanz@fli.de (J.G.); claudia.schulz@live.de (C.S.) \\ 2 Research Center for Emerging Infections and Zoonoses, University of Veterinary Medicine Hannover, \\ Bünteweg 17, 30559 Hanover, Germany \\ 3 Parasitology Unit, University of Hohenheim, Emil-Wolff-Straße 34, 70599 Stuttgart, Germany; \\ Malena1Bestehorn@bundeswehr.org (M.B.); GerhardDobler@bundeswehr.org (G.D.) \\ 4 Institute of Microbiology of the Bundeswehr, Neuherbergstraße 11, 80937 Munich, Germany \\ 5 The Governmental Institute of Public Health of Lower Saxony (NLGA), Roesebeckstraße 4-6, \\ 30449 Hannover, Germany; parasitologie@nlga.Niedersachsen.de (B.M.); \\ Masyar.Monazahian@nlga.Niedersachsen.de (M.M.) \\ * Correspondence: stefanie.becker@tiho-hannover.de; Tel.: +49-511-953-6112 \\ + These authors contributed equally to this work.
}

Received: 15 March 2019; Accepted: 18 May 2019; Published: 21 May 2019

\begin{abstract}
Tick-borne encephalitis (TBE) is the most important tick-borne arboviral disease in Europe. Presently, the main endemic regions in Germany are located in the southern half of the country. Although recently, sporadic human TBE cases were reported outside of these known endemic regions. The detection and characterization of invading TBE virus (TBEV) strains will considerably facilitate the surveillance and assessment of this important disease. In 2018, ticks were collected by flagging in several locations of the German federal state of Lower Saxony where TBEV-infections in humans (diagnosed clinical TBE disease or detection of TBEV antibodies) were reported previously. Ticks were pooled according to their developmental stage and tested for TBEV-RNA by RT-qPCR. Five of $730(0.68 \%)$ pools from Ixodes spp. ticks collected in the areas of "Rauher Busch" and "Barsinghausen/Mooshuette" were found positive for TBEV-RNA. Phylogenetic analysis of the whole genomes and E gene sequences revealed a close relationship between the two TBEV isolates, which cluster with a TBEV strain from Poland isolated in 1971. This study provides first data on the phylogeny of TBEV in the German federal state of Lower Saxony, outside of the known TBE endemic areas of Germany. Our results support the hypothesis of an east-west invasion of TBEV strains in Western Europe.
\end{abstract}

Keywords: tick-borne encephalitis virus; tick; phylogenetic analysis; surveillance; field study

\section{Introduction}

Tick-borne encephalitis (TBE) is one of the most important zoonotic central nervous system (CNS) diseases in humans in Europe and Asia with 10,000 to 15,000 cases per year [1]. TBE in humans can result in severe neurological symptoms such as meningitis $(60 \%)$, meningoencephalitis $(30 \%)$ and meningoencephalomyelitis $(10 \%)$ with potential lethal $(0.2 \%-1 \%)$ outcome of the disease $[2,3]$. 
The causative agent, TBE virus (TBEV) belongs to the genus Flavivirus in the Flaviviridae family. Humans generally become infected with TBEV following the bite of an infected tick. Mainly in eastern European countries, the alimentary transmission of the virus via contaminated dairy products is another possible cause of TBEV-infection. TBE viruses are divided into five subtypes, whose distribution is geographically linked to the occurrence of their main vector tick species: the European subtype (transmitted mainly by Ixodes ricinus) and the Siberian and Far-Eastern subtypes (both transmitted mainly by I. persulcatus) and two new subtypes, the Baikalian (isolated from I. persulcatus) and the Himalayan subtype (tick vector unknown) [4-7]. To date, only strains of the European subtype have been identified in Germany [8]. The vector tick for TBEV in Germany is the castor bean tick I. ricinus. The sylvatic cycle of TBEV, involves ticks and wild vertebrate hosts, in particular small rodents $[1,9]$. Ticks play the most important role for maintaining TBEV in nature acting both as vector and as reservoir. Due to the short viremia in humans and the absence of detectable virus in cerebrospinal fluid of patients, most TBEV isolates are obtained from questing ticks in so-called TBEV foci [9].

The TBEV genome consists of a positive single-stranded RNA molecule (+ssRNA) of approximately 11 kilobases in length containing one open reading frame (ORF) and encoding for a large polyprotein (about 3400 amino acids) [1]. This polyprotein is cleaved into three structural proteins (C = capsid, $M=$ membrane and $E=$ envelope) and seven non-structural proteins (NS1, NS2A, NS2B, NS3, NS4A, NS4B, NS5), which are necessary for virus replication [10]. The E protein acts as the major surface protein of the virus interacting with cell receptors of tick vectors and mammalian hosts and mediating fusion of the virus with the cell membrane. The virus neutralizing immune response in the mammalian host via antibodies is mediated against the E protein [11].

In Germany, most endemic areas are located in the southern federal states of Bavaria and Baden-Wuerttemberg [12]. Sporadic human TBEV cases have also been reported outside of these endemic areas, e.g., the federal state of Lower Saxony [13]. Since TBE was classified as a notifiable disease in Germany in 2001, 61 human cases have been reported (2001-2018) in the federal state of Lower Saxony [14] (see Figure S1 and Table S1). Detection and characterization of invading TBEV strains will add information to our knowledge on the distribution and spread of TBEV and herewith facilitate the surveillance and understanding of this important disease. Here, we describe the detection, isolation and first phylogenetic data of TBEV strains detected in ticks in the German federal state of Lower Saxony.

\section{Materials and Methods}

\subsection{Virus Screening}

In 2018, 4798 questing ticks (704 adults, 4094 nymphs) were collected by flagging the low vegetation at five different sampling sites located at two distinct areas in Lower Saxony, namely "Rauher Busch" $\left(\mathrm{N} 52^{\circ} 34^{\prime} ; \mathrm{E} 8^{\circ} 48^{\prime}\right)$ and "Barsinghausen/Mooshuette" (N52 $\left.19^{\prime} ; \mathrm{E}^{\circ}{ }^{\circ} 3^{\prime}\right)$. Both locations had been associated with humans that showed clinical TBE disease and/or were seropositive for TBEV. Ticks were pooled according to stage and sampling site and stored at $4{ }^{\circ} \mathrm{C}$ for a maximum of 7 days until further processing. Each of the analyzed pools contained 5 to 10 adults or 10 to 20 nymph ticks. Pools were homogenized using steel beads in $500 \mu \mathrm{L}$ cell culture medium (Leibowitz's L-15 or MEM Eagle, Thermo Scientific, Waltham, MA, USA) and TissueLyser II (Qiagen, Hilden, Germany). The homogenates were clarified by centrifugation and total RNA was extracted from the homogenates using the NucleoSpin ${ }^{\circledR}$ RNA Virus kit (Macherey-Nagel, Dueren, Germany) or MagNA Pure96 Viral RNA Small Volume Kit (Roche, Mannheim, Germany) according to the manufacturer's instructions. The extracted RNA was re-suspended in RNase-free water and screened for TBEV-RNA by quantitative reverse transcription-PCR (RT-qPCR) following the protocol developed by Schwaiger and Cassinotti [15]. TBEV-RNA of the Austrian Neudoerfl strain (U27495.1) was used as a positive control, while RNase-free water served as negative control. Furthermore, the quality of RNA extraction was tested in randomly 
selected samples (10\% of all pools) using a RT-qPCR targeting the $16 \mathrm{~S}$ ribosomal RNA (rRNA) of I. ricinus (Schwaiger and Cassinotti [12]).

\subsection{Virus Cultivation}

We inoculated A549 cells (ATCC ${ }^{\circledR}$ CCL-185 ${ }^{\mathrm{TM}}$ ) with $100 \mu \mathrm{L}$ aliquots of TBEV-RNA positive tick homogenate (diluted 1:10 in minimal essential medium (MEM)). After $1 \mathrm{~h}$ of incubation at $37^{\circ} \mathrm{C}$, the virus homogenates were discarded, cells were washed three times with PBS and $4 \mathrm{~mL}$ of MEM supplemented with $2 \%$ fetal bovine serum (FBS) and antibiotics (Penicillin/Streptomycin Pan Biotech; Aidenbach, Germany, Gentamicin/Amphotericin Thermo Fisher, Waltham, MA, USA) were added. Negative controls were inoculated with MEM. This procedure was performed for all five TBEV-RNA positive pools. The homogenates TBEV-LS-Rauher Busch P16, -Rauher Busch P19 and TBEV-LS-Barsinghausen/Mooshuette P51 were done in Hannover at the laboratory of the Research Center for Emerging Infections and Zoonoses, the isolates TBEV-LS-Barsinghausen/Mooshuette-HB IF06 8040 and TBEV-LS-Barsinghausen/Mooshuette-HB IF06 8033 were generated at the laboratory of the Bundeswehr Institute of Microbiology in Munich.

\subsection{PCR and Phylogenetic Analysis of TBEV Isolates}

For the TBEV isolates TBEV-LS-Barsinghausen/Mooshuette-HB IF06 8040 and -HBIF06 8033, and TBEV-LS-Rauher Busch P19 the whole genome was generated. Therefore, two 5.6. kb PCR fragments, covering the whole genome, were generated using primers TBEw-1/TBEw-c5670/TBEw-5451/ TBEw-c11141 of Andersen et al. [16] in a conventional RT-PCR (SuperScript ${ }^{\mathrm{TM}}$ III Reverse Transcriptase; Invitrogen, Karlsruhe, Germany). The PCR products were mechanically sheered using a Bioruptor UCD-200 (Diagenode Diagnostics, Liège, Belgium) and prepared for sequencing using the TruSeq Nano DNA Low Throughput Library Prep Kit (Illumina, Inc., San Diego, CA, USA). The Illumina MiSeq platform was used for sequencing and the sequencing chemistry used was the MiSeq reagent kit V3 (Illumina, Inc., San Diego, CA, USA), according to the manufacture's protocol. De novo assembly of the sequences was performed using the software Spades v.3.12 [17]. The maximum likelihood tree (Figure 1) was generated using the general time reversible model with five categories. The G+ parameter used was 0.3942. The neighbor joining tree (not shown) was calculated using the initial parameters from MEGA6.0 (https://www.megasoftware.net). For statistical support, 1000 bootstraps were calculated for each tree.

A $1488 \mathrm{bp}$ fragment of the E gene was amplified from the different TBEV isolates using the primer pairs TBE-885/TBE-c2571a/b of Kupča et al. [5] and a conventional RT-PCR kit (SuperScript ${ }^{\mathrm{TM}}$ III Reverse Transcriptase; Invitrogen, Karlsruhe, Germany) according to the manufacturer's instructions. Sanger sequencing of the resulting PCR fragment was conducted using the TBE-885/TBE-c2571a/b primers as well as the TBE-c1648 primer [5] (GATC Biotech Ag, Konstanz, Germany). E gene sequences were obtained by de novo assembly of the chromatogram files using Geneious 9.1.5 (https:/www.gene ious.com). The sequences of the E genes for TBEV-LS-Rauher Busch-P16 (MK903683), -P19 (MK903681) and TBEV-LS-Barsinghausen/Mooshuette-P51 (MK903682), -HB IF06 8040 (MK903679) and -HB IF06 8033 (MK903680) as well as available sequences of the E gene from the NCBI GenBank database (http://www.ncbi.nlm.nih.gov/blast/Blast.cgi) and so far unpublished sequences of the German National Reference Laboratory (TBEV Jaegerhof JH98/13 (MK903684); TBEV-RG18-1971-POL (MK903685)) were used for phylogenetic analysis with the MEGA6.0 software (https://www.megasoftware.net). 


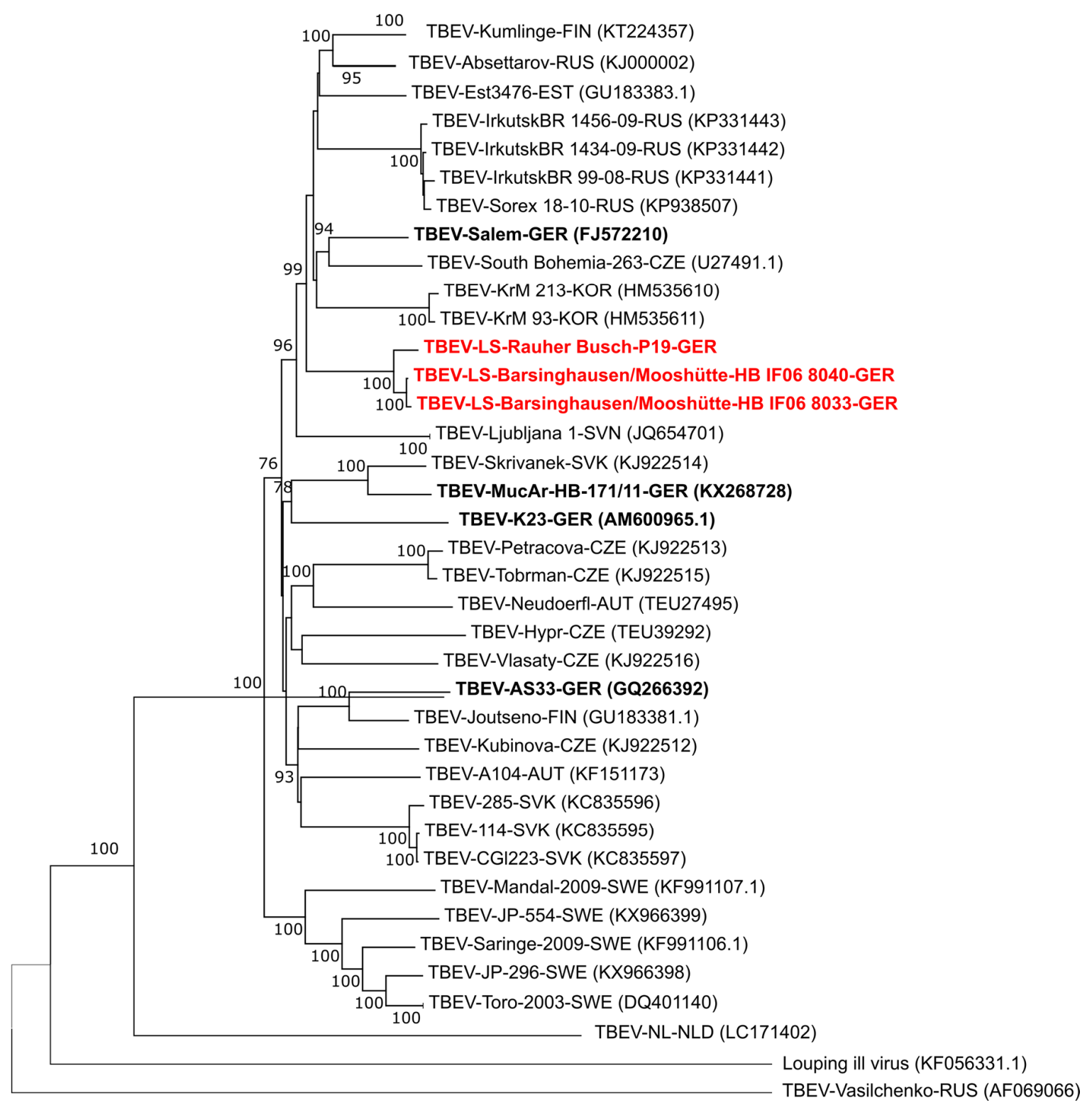

Figure 1. Phylogenetic tree of whole genome sequences available in GenBank and the two TBEV-LS-Barsinghausen/Mooshuette isolates HB IF06 8033 (MK922615) and 8040 (MK922616) and TBEV-LS-Rauher Busch P19 (MK922617) obtained in the present study (marked red). Virus isolate names are given as follows: TBEV-isolate name or number-three letter country code according to UN standard (accession number for the sequences as available from NCBI). UN country codes are: AUT: Austria; CHE: Switzerland; CZE: Czech Republic; EST: Estonia; FIN: Finland; GER: Germany; KOR: Korea; NLD: Netherlands; RUS: Russian Federation; SVN: Slovenia; SVK: Slovakia; SWE: Sweden. All sequences obtained from German isolates are marked bold, the new sequences obtained during this study are additionally marked in red. As an outgroup the Louping ill virus (KF056331.1) and TBEV-Vasilchenko (AF069066) were used.

\section{Results and Discussion}

Of the 730 tested pools, we found five pools (0.68\%) positive for TBEV-RNA. At the collection site "Rauher Busch", two pools with adult ticks and at the site of "Barsinghausen/Mooshuette" one and two pools with adults and nymphs, respectively, were positive for TBEV-RNA. The amplification of 16S rRNA was successful in all tested pools indicating efficient RNA extraction and minimizing the probability of false negative results due to inhibition of the PCR reaction by tick products. The minimum 
infection rates (MIR) for TBEV in ticks from Lower Saxony were $1.05 \%$ in adults and $0.45 \%$ in nymphs, which is similar to the MIR for TBEV described in Sweden in 2008 (0.55\% in adults and $0.23 \%$ in nymphs) [18], Poland and Lithuania in 2006-2009 (0.24\% in adults and 0.11\% in nymphs) [19] and in the district of Passau in Bavaria, Germany, in $2001(0.03 \%-6.38 \%$ in adults and $0.08 \%-1.1 \%$ in nymphs) [20] and 1997-1998 (0.9\%-2.0\% for all tick stages) [21]. The MIR of nymph and adult tick stages from endemic foci in the Black Forest region near Freiburg in Baden-Wuerttemberg, Germany, were slightly higher in 1997 (2.9\%-3.4\%) and in 1998 (0.6\%-1.1\%) [21]. Similarly, a MIR of 4.48\% in adults and $0.51 \%$ in nymphs was reported at the well described TBEV focus Torö island, south-east of Stockholm, in 2008 [18]. These data suggest that TBEV prevalence considerably varies by year and location, most probably due to small sample sizes analyzed and due to the frequency of sampled individual tick stages [22]. However, our MIR in Lower Saxony lies well within the published range.

To confirm the presence of infective virus from the tick samples, all homogenates were inoculated on A549 cells. The three homogenates TBEV-LS-Rauher Busch P16 and 19, and TBEV-LSBarsinghausen/Mooshuette P51 done at the Research Center for Emerging Infections and Zoonoses caused a cytopathic effect (CPE) beginning 3-4 days post infection (p.i.). RT-qPCR analyses of the cell culture supernatants collected at 6-7 days p.i. revealed positive results for TBEV-RNA in the samples TBEV-LS-Rauher Busch-P16, TBEV-LS-Rauher Busch-P19 and TBEV-LS-Barsinghausen/Mooshuette-P51. Hereinafter, these three virus isolates are referred to as TBEV-LS-Rauher Busch-P16, TBEV-LS-Rauher Busch-P19 and TBEV-LS-Barsinghausen/Mooshuette-P51. Two additional viruses that were isolated at the Bundeswehr Institute of Microbiology in Munich are referred to as TBEV-LS-Barsinghausen/ Mooshuette-HB IF06 8040 and TBEV-LS-Barsinghausen/Mooshuette-HB IF06 8033.

The genome sequences obtained during this study (TBEV-LS-Barsinghausen/Mooshuette isolates HB IF06 8033 (MK922615) and 8040 (MK922616) and TBEV-LS-Rauher Busch P19 (MK922617)) were used for the construction of a phylogenetic tree using the complete open reading frame of these and all available sequences from NCBI GenBank (Figure 1).

Based on the full genome sequences, the new isolates TBEV-LS-Rauher Busch P19 and TBEV-LS-Barsinghausen/Mooshuette are closely related to each other and more distantly related to other German isolates where full genome sequences are available such as Salem, K23, MucAr and AS33. The closest relationship is seen to a cluster including Russian, Korean, Finnish, South Bohemian and the Salem sequences. In addition, we compared the sequences of the open reading frame of the two isolates TBEV-LS- Rauher Busch P19 and TBEV-LS-Barsinghausen/Mooshuette HBIF06 8033 to each other (Table S2). This comparison revealed 36 nucleotide difference between the two sequences resulting in 10 amino acid exchanges. This again points towards a close genetic relationship of these isolates as compared to the study by Kupča et al. [5] which describes the relationship of the isolate AS33 and Salem showing 251 nucleotide differences resulting in 26 amino acid exchanges between those two strains.

For a further in-depth characterization of the virus isolates TBEV-LS-Rauher Busch-P16 and -P19 as well as TBEV-LS-Barsinghausen/Mooshuette-P51 HB IF06 8040 and HB IF06 8033, we used a PCR assay described by Kupča et al. [5] to amplify the viral E gene. The E gene is the most commonly used marker for phylogenetic classification of TBEV subtypes and strains and most sequence data are available for this gene sequence [23]. The maximum likelihood and neighbor joining trees generated by phylogenetic analysis for the E gene (Figure 2) compared with the respective trees of the whole coding sequences (Figure 1) resulted in a similar dendrogram. The close genetic relationship of the E gene sequences from TBEV isolates in Lower Saxony Barsinghausen/Mooshuette and Rauher Busch despite geographic distance between those two sampling locations indicate that the two isolates have a common ancestor and have spread locally. This is unusual as two natural TBE foci separated by a distance of about $60 \mathrm{~km}$ usually have genetically more differentiated viruses [24]. The close genetic relationship implies that the TBEV strains were recently distributed from one location to the other, and so far had no time to adapt and evolve in a focus-specific TBE genetic clade. 


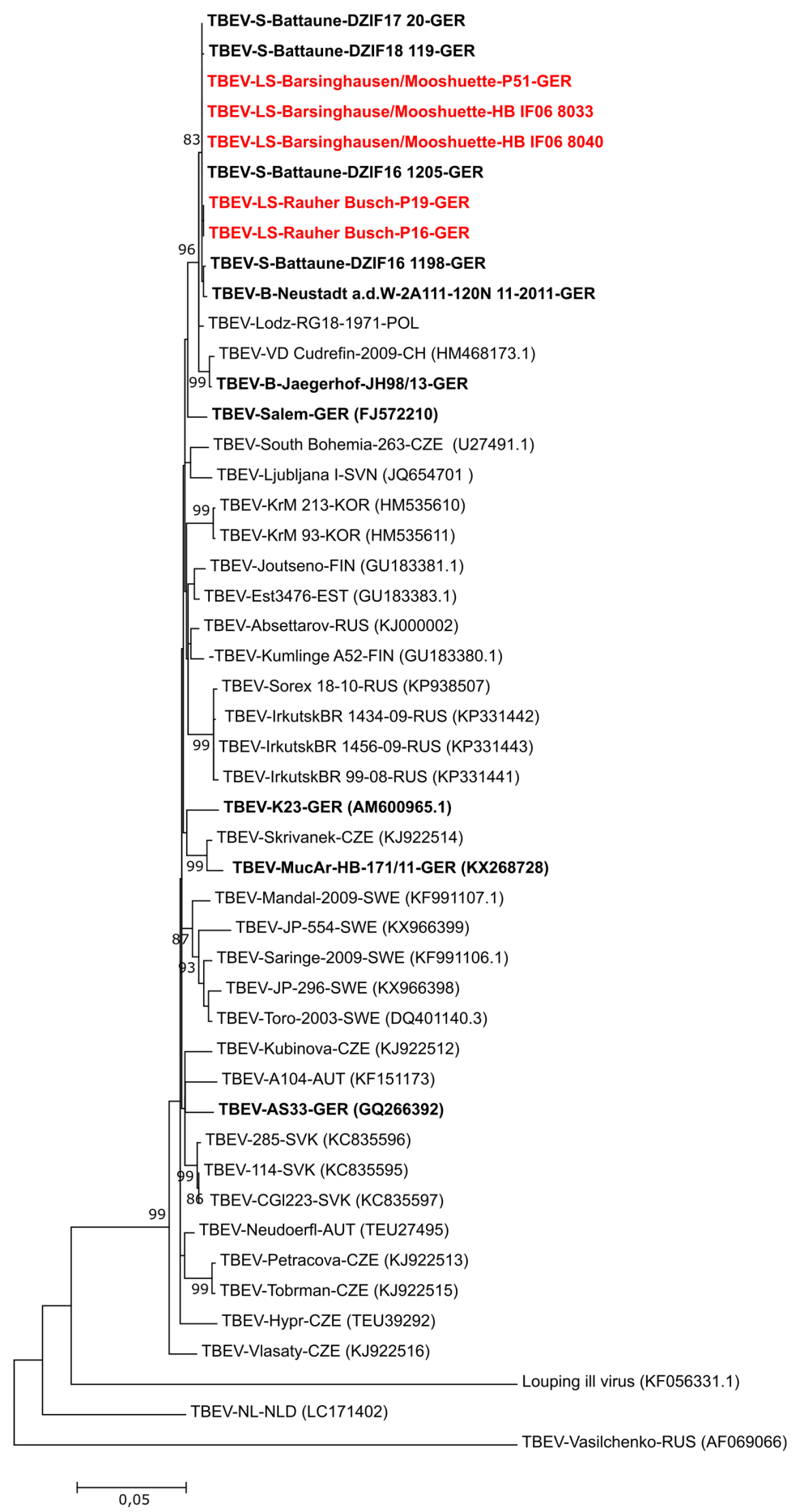

Figure 2. Phylogenetic tree (Maximum likelihood Tree, MEGA6.0) of the five "TBEV-LS" isolates collected in "Barsinghausen/Mooshuette" and "Rauher Busch" (TBEV-LS-Rauher Busch-P16 (MK903683), -P19 (MK903681) and TBEV-LS-Barsinghausen/Mooshuette-P51 (MK903682), -HB IF06 8040 (MK903679) and -HB IF06 8033 (MK903680) in the German federal state of Lower Saxony, 2018, using E gene sequences. Virus isolate names are given as follows: TBEV-isolate name or number-three letter country code according to UN standard (accession number of the sequence as available from NCBI). UN country codes are: AUT: Austria; CHE: Switzerland; CZE: Czech Republic; EST: Estonia; FIN: Finland; GER: Germany; KOR: Korea; NLD: Netherlands; POL: Poland; RUS: Russian Federation; SVN: Slovenia; SVK: Slovakia; SWE: Sweden. As an outgroup strain Louping ill virus (KF056331.1), TBEV-NL (LC171402) and TBEV-Vasilchenko (AF069066) were used. All sequences obtained from German isolates are marked bold, the new sequences obtained during this study are additionally marked in red. 
Furthermore, the E gene sequences of TBEV-LS-Rauher Busch-P16 and -P19 as well as TBEV-LS-Barsinghausen/Mooshuette-P51, -HB IF06 8040 and -HB IF06 8033 clustered with TBEV strains from Battaune in the German federal state of Saxony (TBEV-S-Battaune-DZIF16 1198; DZIF16 1205, DZIF17 20 and DZIF18 119) [25] and Lodz in Poland (kindly provided by Pawel Steffanoff) (Figure 2). The Polish isolate was detected in 1971 and might therefore be an ancestor for the Battaune and the Lower Saxony isolates. The proposed east-west spread is in accordance with one of the three main hypotheses for TBEV spread. According to molecular phylogenetic data of E gene sequences, TBEV appeared around 2500 years ago in the Far East and then spread to West Eurasia $[23,24,26]$. This hypothesis is however contrasted by other studies. For example, analyses of whole genome sequences suggest Western Siberia as an origin for TBEV and a subsequent bi-directional spread of TBEV to West and East Eurasia [27]. Nevertheless, on the smaller regional scale, the general east-west concept is supported by all published data collected so far $[24,26,28]$.

Regarding the means of TBEV spread, there are several possibilities: (i) continuous spread: terrestrial transport of TBEV-infected ticks by infestation on wild animal hosts such as roe deer or wild boar [29,30], (ii) discontinuous spread: aerial transport of TBEV-infected ticks by avian hosts, mostly associated with stepping stones of migratory bird species [31], or anthropogenic transport including transport of infected animals [22]. For the recent TBEV isolates from the upper Rhine valley detected in 2016-2018, terrestrial and aerial spreading pattern by wild animals seem to apply [32]. Weidmann et al. [24] discussed discontinues spread of TBEV via anthropogenic routes to explain the phylogenetic relationship between TBEV isolates from the Czech Republic (South Moravia) and Bavaria (Haselmühl) in Germany. The two Czech and German regions where the respective TBEV isolates were detected are separated by a mountain range of $1400 \mathrm{~m}$ altitude; hence, making TBEV-spread by terrestrial hosts rather unlikely. Similarly, the close phylogenetic relationship between the TBEV-LS-Barsinghausen/Mooshuette/Rauher Busch and TBEV-S-Battaune strains with the TBEV-Lodz-RG18-1971-Pol strain from Lodz in Poland (a distance of $750 \mathrm{~km}$ via the E30 and A2 motorways or of $870 \mathrm{~km}$ via the E40 and A4 motorways in Poland and Germany, respectively; see Figure S2) might also reflect a direct transportation of TBEV-infected ticks between both locations by motorized vehicles. The E30 and E40 motorways in Poland are highly trafficked by trucks for freight transports between Germany and Poland. In 2014, these two motorways were the second most important routes for goods transferred to Germany with an annual volume of 25 million tons [33]. Interestingly, the second route via the E40 passes by Battaune in Saxony - thus, supporting the hypothesis of a common introduction route for TBEV-LS-Barsinghausen/Mooshuette/Rauher Busch and TBEV-S-Battaune strains (Figure S2). In a Russian study it has been hypothesized that the Baltic TBEV strains of the Siberian and Far Eastern subtypes were exported from their Russian areas of distribution in Siberia along the routes of the trans Baikalian highway and the trans Baikalian railway [34]. However, the currently available data do not allow final conclusions on the origin of the TBEV strains from Lower Saxony. More isolates from other locations along the E30 or E40 would be needed to either confirm or disprove the hypothesis of freight transport as an anthropogenic route for TBEV distribution.

Taken together, the detection of five different TBEV strains from two different locations in Lower Saxony highlights the increased prevalence of this virus in German regions that have been formally classified as non-endemic for TBEV. Accordingly, the increased TBEV prevalence demands an increase of public awareness raising and surveillance efforts for TBEV in Lower Saxony and possibly the other northern federal states of Germany. Furthermore, the first phylogenetic characterization of TBEV strains from Lower Saxony suggests a classical east-west invasion event as indicated by the high similarity of virus strains from Saxony and Lower Saxony, supporting the existing hypothesis of TBEV distribution patterns.

Supplementary Materials: The following are available online at http://www.mdpi.com/1999-4915/11/5/462/s1, Figure S1: TBE case numbers in all German federal states in the years 2001-2019; Figure S2: Map of central Europe with all TBEV isolation sites of TBEV strains used for full genome and E gene analysis. Table S1: Table S1: Case numbers in all German federal states in the years 2001-2019; Table S2: Sequence comparison of the isolates TBEV-LS-Rauher Busch P19 and TBEV-LS-Barsinghausen/Mooshuette HB IF06 8033. 
Author Contributions: S.C.B., M.B. (Mathias Boelke), M.M. and G.D. conceived and designed the experiments. M.B. (Mathias Boelke), M.B. (Malena Bestehorn), B.M., M.K., K.L., J.G., and C.S. collected and analyzed the samples, M.B. (Malena Bestehorn) and M.B. (Mathias Boelke) performed the formal analysis, S.C.B., M.B. (Mathias Boelke), M.B. (Malena Bestehorn) and G.D. wrote the original draft.; S.C.B., C.S. G.D. and M.M. reviewed and edited the draft, S.C.B. and G.D. participated in funding acquisition.

Funding: This research was funded by DFG grant number BE 5748/4-1 and the TBENAGER consortium FKZ 01KI1728F and 01KI1728A. This publication was supported by Deutsche Forschungsgemeinschaft and University of Veterinary Medicine Hannover, Foundation within the funding programme Open Access Publishing.

Acknowledgments: We would like to thank Hella Heidtmann for her excellent technical assistance and her enormous support conducting this work.

Conflicts of Interest: The authors declare no conflict of interest. The funders had no role in the design of the study; in the collection, analyses, or interpretation of data; in the writing of the manuscript, or in the decision to publish the results.

\section{References}

1. Gritsun, T.S.; Lashkevich, V.A.; Gould, E.A. Tick-borne encephalitis. Antivir. Res. 2003, 57, 129-146. [CrossRef]

2. Lindquist, L.; Vapalahti, O. Tick-borne encephalitis. Lancet 2008, 371, 1861-1871. [CrossRef]

3. Hayasaka, D.; Nagata, N.; Fujii, Y.; Hasegawa, H.; Sata, T.; Suzuki, R.; Gould, E.A.; Takashima, I.; Koike, S. Mortality following peripheral infection with Tick-borne encephalitis virus results from a combination of central nervous system pathology, systemic inflammatory and stress responses. Virology 2009, 390, 139-150. [CrossRef] [PubMed]

4. Heinz, F.X. Tick-borne encephalitis virus: Advances in molecular biology and vaccination strategy in the next century. Zent. Bakteriol. 1999, 289, 506-510. [CrossRef]

5. Kupča, A.M.; Essbauer, S.; Zoeller, G.; de Mendonça, P.G.; Brey, R.; Rinder, M.; Pfister, K.; Spiegel, M.; Doerrbecker, B.; Pfeffer, M.; et al. Isolation and molecular characterization of a tick-borne encephalitis virus strain from a new tick-borne encephalitis focus with severe cases in Bavaria, Germany. Ticks Tick-Borne Dis. 2010, 1, 44-51. [CrossRef]

6. Dai, X.; Shang, G.; Lu, S.; Yang, J.; Xu, J. A new subtype of eastern tick-borne encephalitis virus discovered in Qinghai-Tibet Plateau, China. Emerg. Microbes Infect. 2018, 7, 74. [CrossRef]

7. Kovalev, S.Y.; Mukhacheva, T.A. Reconsidering the classification of tick-borne encephalitis virus within the Siberian subtype gives new insights into its evolutionary history. Infect. Genet. Evol. 2017, 55, 159-165. [CrossRef] [PubMed]

8. Suss, J. Tick-borne encephalitis in Europe and beyond-the epidemiological situation as of 2007. Euro Surveill. 2008, 13, 717-727.

9. Charrel, R.N.; Attoui, H.; Butenko, A.M.; Clegg, J.C.; Deubel, V.; Frolova, T.V.; Gould, E.A.; Gritsun, T.S.; Heinz, F.X.; Labuda, M.; et al. Tick-borne virus diseases of human interest in Europe. Clin. Microbiol. Infect. 2004, 10, 1040-1055. [CrossRef]

10. Mandl, C.W.; Heinz, F.X.; Stöckl, E.; Kunz, C. Genome sequence of tick-borne encephalitis virus (Western subtype) and comparative analysis of nonstructural proteins with other flaviviruses. Virology 1989, 173, 291-301. [CrossRef]

11. Heinz, F.X. Epitope mapping of flavivirus glycoproteins. Adv. Virus Res. 1986, 31, 103-168. [PubMed]

12. Epidemiologisches Bulletin 17/2018. In Robert Koch Institut. 2018, Vol. 17. Available online: https://www.rki.de /DE/Content/Infekt/EpidBull/Archiv/2018/Ausgaben/17_18.html (accessed on 15 March 2019).

13. Dobler, G.; Gniel, D.; Petermann, R.; Pfeffer, M. Epidemiology and distribution of tick-borne encephalitis. Wien. Med. Wochenschr. 2012, 162, 230-238. [CrossRef]

14. SurvStat@RKI 2.0. In Robert Koch Institut. 2018. Available online: https://survstat.rki.de/Content/Instructio n/Main.aspx (accessed on 15 March 2019).

15. Schwaiger, M.; Cassinotti, P. Development of a quantitative real-time RT-PCR assay with internal control for the laboratory detection of tick borne encephalitis virus (TBEV) RNA. J. Clin. Virol. 2003, 27, 136-145. [CrossRef]

16. Andersen, N.S.; Bestehorn, M.; Chitimia-Dobler, L.; Kolmos, H.J.; Jensen, P.M.; Dobler, G.; Skarphédinsson, S. Phylogenetic characterization of tick-borne encephalitis virus from Bornholm, Denmark. Ticks Tick-Borne Dis. 2018, 10, 533-539. [CrossRef] [PubMed] 
17. Nurk, S.; Bankevich, A.; Antipov, D.; Gurevich, A.; Korobeynikov, A.; Lapidus, A.; Prjibelsky, A.; Pyshkin, A.; Sirotkin, A.; Sirotkin, Y.; et al. Assembling Genomes and Mini-metagenomes from Highly Chimeric Reads, Berlin, Heidelberg, 2013; Springer: Berlin, Heidelberg, 2013; pp. 158-170.

18. Pettersson, J.H.; Golovljova, I.; Vene, S.; Jaenson, T.G. Prevalence of tick-borne encephalitis virus in Ixodes ricinus ticks in northern Europe with particular reference to Southern Sweden. Parasites Vectors 2014, 7, 102. [CrossRef] [PubMed]

19. Katargina, O.; Russakova, S.; Geller, J.; Kondrusik, M.; Zajkowska, J.; Zygutiene, M.; Bormane, A.; Trofimova, J.; Golovljova, I. Detection and characterization of tick-borne encephalitis virus in Baltic countries and eastern Poland. Plos ONE 2013, 8, e61374. [CrossRef]

20. Süss, J.; Schrader, C.; Falk, U.; Wohanka, N. Tick-borne encephalitis (TBE) in Germany — Epidemiological data, development of risk areas and virus prevalence in field-collected ticks and in ticks removed from humans. Int. J. Med. Microbiol. Suppl. 2004, 293, 69-79. [CrossRef]

21. Suss, J.; Schrader, C.; Abel, U.; Voigt, W.P.; Schosser, R. Annual and seasonal variation of tick-borne encephalitis virus (TBEV) prevalence in ticks in selected hot spot areas in Germany using a nRT-PCR: results from 1997 and 1998. Zent. Bakteriol. 1999, 289, 564-578. [CrossRef]

22. Jovani, R.; Tella, J.L. Parasite prevalence and sample size: Misconceptions and solutions. Trends Parasitol. 2006, 22, 214-218. [CrossRef]

23. Kovalev, S.Y.; Mukhacheva, T.A. Tick-borne encephalitis virus subtypes emerged through rapid vector switches rather than gradual evolution. Ecol. Evol. 2014, 4, 4307-4316. [CrossRef] [PubMed]

24. Weidmann, M.; Růžek, D.; Křivanec, K.; Zöller, G.; Essbauer, S.; Pfeffer, M.; Zanotto, P.M.d.A.; Hufert, F.T.; Dobler, G. Relation of genetic phylogeny and geographical distance of tick-borne encephalitis virus in central Europe. J. Gen. Virol. 2011, 92, 1906-1916. [CrossRef]

25. Chitimia-Dobler, L.; Lemhöfer, G.; Król, N.; Bestehorn, M.; Dobler, G.; Pfeffer, M. Repeated isolation of tick-borne encephalitis virus from adult Dermacentor reticulatus ticks in an endemic area in Germany. Parasites Vectors 2019, 12, 90. [CrossRef]

26. Zanotto, P.M.; Gao, G.F.; Gritsun, T.; Marin, M.S.; Jiang, W.R.; Venugopal, K.; Reid, H.W.; Gould, E.A. An arbovirus cline across the northern hemisphere. Virology 1995, 210, 152-159. [CrossRef] [PubMed]

27. Heinze, D.M.; Gould, E.A.; Forrester, N.L. Revisiting the clinal concept of evolution and dispersal for the tick-borne flaviviruses by using phylogenetic and biogeographic analyses. J. Virol. 2012, 86, 8663-8671. [CrossRef]

28. Weidmann, M.; Frey, S.; Freire, C.C.; Essbauer, S.; Ruzek, D.; Klempa, B.; Zubrikova, D.; Vogerl, M.; Pfeffer, M.; Hufert, F.T; et al. Molecular phylogeography of tick-borne encephalitis virus in central Europe. J. Gen. Virol. 2013, 94 (Pt 9), 2129-2139. [CrossRef]

29. Kriz, B.; Daniel, M.; Benes, C.; Maly, M. The role of game (wild boar and roe deer) in the spread of tick-borne encephalitis in the Czech Republic. Vector Borne Zoonotic Dis. (Larchmt. N.Y.) 2014, 14, 801-807. [CrossRef] [PubMed]

30. Balling, A.; Plessow, U.; Beer, M.; Pfeffer, M. Prevalence of antibodies against tick-borne encephalitis virus in wild game from Saxony, Germany. Ticks Tick-Borne Dis. 2014, 5, 805-809. [CrossRef]

31. Waldenstrom, J.; Lundkvist, A.; Falk, K.I.; Garpmo, U.; Bergstrom, S.; Lindegren, G.; Sjostedt, A.; Mejlon, H.; Fransson, T.; Haemig, P.D.; et al. Migrating birds and tickborne encephalitis virus. Emerg. Infect. Dis. 2007, 13, 1215-1218. [CrossRef]

32. Bestehorn, M.; Weigold, S.; Kern, W.V.; Chitimia-Dobler, L.; Mackenstedt, U.; Dobler, G.; Borde, J.P. Phylogenetics of tick-borne encephalitis virus in endemic foci in the upper Rhine region in France and Germany. PLoS ONE 2018, 13, e0204790. [CrossRef] [PubMed]

33. Hütter, A. Güterverkehr in Deutschland 2014. In Statistisches Bundesamt Wista; 2016; Vol. WISTA 1/2016, pp. 47-62. Available online: https://www.destatis.de/DE/Publikationen/WirtschaftStatistik/2016/01/Wista_1 2016.pdf?_blob=publicationFile (accessed on 15 March 2019).

34. Kovalev, S.Y.; Mukhacheva, T.A. Clusterons as a tool for monitoring populations of tick-borne encephalitis virus. J. Med. Virol. 2014, 86, 283-289. [CrossRef]

(C) 2019 by the authors. Licensee MDPI, Basel, Switzerland. This article is an open access article distributed under the terms and conditions of the Creative Commons Attribution (CC BY) license (http://creativecommons.org/licenses/by/4.0/). 